\title{
Poisoning in children 5: Rare and dangerous poisons
}

\section{Riordan, G Rylance, K Berry}

Management of children who have ingested $\beta$ blockers, digoxin, oral hypoglycaemics, organophosphates, carbon monoxide, cyanide, isopropanol, ethylene glycol, methanol, Ecstasy, LSD, cocaine, nicotine, and isoniazid

I the final paper in this series of articles on the management of poisonIng, we deal with exposures to a variety of rare, but potentially very dangerous, toxins.

\section{$\beta$ BLOCKERS}

$\beta$ Blockers competitively antagonise the binding of catecholamines to $\beta$ receptors. The effect of specific agents in overdose depends on their receptor specificity, lipid solubility, partial agonist activity, and dose.

Bradycardia and hypotension are the commonest signs of cardiovascular toxicity, but tachycardia and hypertension can occur if a partial agonist is consumed. Other signs of cardiovascular toxicity include varying degrees of heart block, shock, and pulmonary oedema. Central effects can occur, particularly with propanolol, and include lethargy, hallucinations, and convulsions. Hypoglycaemia can also occur.

Asymptomatic children should receive activated charcoal. A period of 12 hours observation is advisable. ${ }^{1}$ Symptomatic children require intensive monitoring. Hypotension may respond to intravenous fluids. In resistant cases, intravenous glucagon $(50-150 \mu \mathrm{g} / \mathrm{kg}$ in $5 \%$ dextrose) is the treatment of choice. High dose glucagon stimulates myocardial adenylate cyclase directly, bypassing $\beta$ receptors. Isoprenaline or cardiac pacing may be required. Regular estimation of blood sugar is essential.

\section{DIGOXIN}

Digoxin is potentially extremely toxic in overdose. Children with underlying cardiac disease are particularly at risk and should always receive treatment. Children without a history of heart disease require treatment if they have ingested more than $100 \mu \mathrm{g} / \mathrm{kg}$ body weight.

The toxic effects of digoxin include nausea, vomiting, hypotension, hyperkalaemia, and a multitude of cardiac arrhythmias. ${ }^{2}$ Careful monitoring is essential as patients can deteriorate suddenly.
Treatment in the first instance is with activated charcoal. Repeated doses should be considered. ${ }^{3}$ Blood pressure and ECG monitoring are required. Electrolytes should be monitored frequently. Hyperkalaemia should be corrected using ion exchange resins, glucose and insulin, or dialysis. Salbutamol and calcium infusions should be avoided because of their potential to destabilise the myocardium.

Bradyarrhythmias may require treatment with atropine or cardiac pacing. Tachyarrhythmias may respond to lignocaine, amiodarone, or phenytoin. ${ }^{14}$ There is an increased risk of inducing asystole with cardioversion and this should be used only as a last resort.

The use of digoxin antibodies should be considered where patients are resistant to supportive treatment or a very large amount of digoxin has been ingested (more than $300 \mu \mathrm{g} / \mathrm{kg}$ ).

Plasma digoxin concentrations can be determined six hours post-ingestion. The upper end of the therapeutic range is $2 \mu \mathrm{g} / \mathrm{l}$. A blood level above $15 \mu \mathrm{g} / \mathrm{l}$ usually indicates severe toxicity. Levels between these points should be interpreted with caution, as they do not correlate accurately with clinical severity.

\section{ORAL HYPOGLYCAEMICS}

Sulphonylurea type drugs produce hypoglycaemia by depolarising pancreatic $\beta$ cells and increasing insulin release. Ingestion of a single tablet can produce symptomatic hypoglycaemia in children. Activated charcoal should be administered. Asymptomatic children should be observed overnight as effects can be delayed for up to 16 hours. ${ }^{5}$ Symptomatic children require intensive monitoring. Hypoglycaemia should be treated with intravenous dextrose infusion. Resistant hypoglycaemia may respond to subcutaneous injection of the somatostatin analogue, octreotide, which inhibits pancreatic insulin release. ${ }^{6}$

Metformin, a biguanide, acts by increasing glucose uptake into the muscles, inhibiting its gastrointestinal uptake and reducing hepatic gluconeogenesis. In overdose, it can produce significant gastrointestinal disturbance. In addition to hypoglycaemia, metformin can induce a profound lactic acidosis. ${ }^{7}$ Serum electrolytes, lactate, and bicarbonate should be monitored. Hypoglycaemia should be treated with intravenous dextrose infusion. Asymptomatic patients should be observed for 12 hours. $^{1}$

Acarbose inhibits glucosidases in the intestine, preventing the digestion and absorption of complex carbohydrates. Hypoglycaemia is unlikely but diarrhoea may occur, particularly if patients receive carbohydrate rich foods following ingestion.

Repaglinide is a newer oral hypoglycaemic agent. It acts by stimulating insulin release but has a very short duration of action. ${ }^{7}$ Blood sugar should be checked and symptomatic children should receive oral glucose. Effects are likely to be short lived.

\section{ORGANOPHOSPHATES}

Organophosphates are found in insecticides, sheep dip, and lice treatments. They produce irreversible acetylcholinesterase inhibition. Accumulation of acetylcholine stimulates muscarinic receptors at parasympathetic postganglionic synapses.

Symptoms of organophosphate poisoning can be delayed for up to 24 hours post-exposure. Symptoms, produced by parasympathetic over stimulation, include increased secretions from salivary, lacrimal, bronchial, and gastrointestinal glands, increased peristaltic activity, bronchoconstriction, bradycardia and hypotension, miosis, and loss of visual acuity. Urinary and faecal incontinence occur secondary to loss of sphincter control. In severe cases, overwhelming tracheobronchial secretions can lead to respiratory compromise. In large doses, organophosphates produce muscle stimulation followed by paralysis because of a depolarising block. Hyperglycaemia and glycosuria without ketonuria may also occur.

While organophosphates can be absorbed via the skin, symptoms rarely result from acute exposure. Ingestion carries the highest risk of acute toxicity.

Asymptomatic children should be observed overnight. Symptomatic children require careful observation. Mild symptoms can be treated with supportive therapy alone. Patients with cardiorespiratory compromise require intensive care. Atropine, often in very high dosage, acts as an antidote-blocking muscarinic receptors and preventing the excessive activity of acetylcholine.

Patients not responding to treatment with atropine should be treated with pralidoxime. This drug reactivates inactivated acetylcholinesterase. Pralidoxime 
Table 1 Antidotes for cyanide poisoning

\begin{tabular}{|c|c|c|c|c|}
\hline Order & Antidote & Dose & Side effects & Comments \\
\hline 1st line & Amyl nitrite & $\begin{array}{l}\text { Crushable perles, vapour inhaled, } \\
\text { either directly or via a bag and } \\
\text { mask }\end{array}$ & $\begin{array}{l}\text { Methaemoglobinaemia } \\
\text { Vasodilatation } \\
\text { Headache }\end{array}$ & $\begin{array}{l}\text { The mechanism of action of amyl nitrite is } \\
\text { unclear but may relate to its action as a } \\
\text { vasodilator }\end{array}$ \\
\hline 1st line & $\begin{array}{l}\text { Sodium } \\
\text { thiosulphate }\end{array}$ & $\begin{array}{l}412.5 \mathrm{mg} / \mathrm{kg}(1.65 \mathrm{ml} / \mathrm{kg} \text { of } \\
25 \% \text { solution), maximum } 50 \mathrm{ml} \text {, } \\
\text { give over } 10 \text { minutes }\end{array}$ & $\begin{array}{l}\text { Nausea } \\
\text { Vomiting } \\
\text { Muscle cramps } \\
\text { Arthralgia }\end{array}$ & $\begin{array}{l}\text { Combines with } \mathrm{CN} \text { to form thiocyanate; } \\
\text { this is non-toxic and excreted in the urine }\end{array}$ \\
\hline 2nd line & Sodium nitrite & $\begin{array}{l}0.33 \mathrm{ml} / \mathrm{kg} \text { of } 3 \% \text { solution, } \\
\text { maximum } 10 \mathrm{ml} \text {, over } 4 \text { minutes }\end{array}$ & $\begin{array}{l}\text { Potentially fatal } \\
\text { methaemoglobinaemia, avoid } \\
\text { excessive dose, keep metHb at } \\
\text { about } 20 \%\end{array}$ & $\begin{array}{l}\text { See amyl nitrite. For moderate to severe } \\
\text { poisoning, do not use as prophylaxis }\end{array}$ \\
\hline 2nd line & Dicobalt EDTA & $\begin{array}{l}4 \mathrm{mg} / \mathrm{kg} \text { over } 1 \text { minute, followed } \\
\text { by } 50 \mathrm{ml} 50 \% \text { dextrose }\end{array}$ & $\begin{array}{l}\text { In the absence of cyanide, cobalt } \\
\text { toxicity can be severe } \\
\text { Anaphylaxis }\end{array}$ & $\begin{array}{l}\text { Chelates cyanide and does not produce } \\
\text { metHb. For moderate to severe poisoning, } \\
\text { do not use as prophylaxis }\end{array}$ \\
\hline
\end{tabular}

is most effective as an antidote when given within the first 24 hours postingestion.

\section{CARBON MONOXIDE}

Carbon monoxide poisoning accounts for the largest group of poisoning deaths among children. ${ }^{8}$

Carbon monoxide (CO) is most frequently encountered as a product of incomplete combustion. The affinity of haemoglobin for carbon monoxide is 210 times its affinity for oxygen. Once bound to haemoglobin, carbon monoxide dissociates very slowly. In addition, the binding of a single CO molecule to haemoglobin increases the strength with which oxygen molecules are attached, making oxygen dissociation in the tissues harder. The amount of carboxyhaemoglobin $(\mathrm{COHb})$ formed depends on the concentration in inspired air and the duration of exposure.

Carbon monoxide dissolved in the plasma acts as a direct cellular poison in its own right. Reacting with other haem proteins, such as mitochondrial cytochromes, it disrupts cellular metabolism.

Patient with up to $20 \%$ of haemoglobin affected complain of headaches and nausea. At $20-40 \%$ involvement, patients tire and become confused. Involvement of more than $40 \%$ haemoglobin results in ataxia, collapse, and coma. Cardiac arrhythmias, cerebral oedema, and acidosis can occur.

The increased affinity of $\mathrm{COHb}$ for oxygen means that haemoglobin molecules become saturated at very low partial pressures of oxygen. Oxygen saturation monitors are therefore very unreliable in the presence of $\mathrm{COHb}-$ saturations of $100 \%$ occurring in the presence of significant hypoxia. Conventional blood gas analysers can also be misleading.

Patients considered at risk of carbon monoxide poisoning should be removed from ongoing exposure. Treatment with as high a concentration of inspired oxygen as circumstances permit should be commenced immediately. High concentrations of oxygen hasten the dissociation of $\mathrm{COHb}$.

The classical sign of "cherry red" lips and nail beds is unusual. Signs of cardiovascular or neurological toxicity should be carefully sought. There are often associated injuries, such as burns or smoke inhalation.

Blood should be taken for acid-base balance, electrolytes, and muscle enzymes. Metabolic acidosis is common and should not be over corrected, as a mild metabolic acidosis will enhance oxygen dissociation in the tissues.

Wherever possible exposure should be confirmed by direct, end tidal measurement of CO in expired air. Where this is not possible, or practicable, spectrophotometric measurement of $\mathrm{COHb}$, from a sample of arterial or venous blood, should be undertaken. The former test is preferable; blood $\mathrm{COHb}$ levels exclude dissolved $\mathrm{CO}$ and can underestimate exposure. Measurements of $\mathrm{CO}$ are best made as soon as possible following exposure; however, the half life of $\mathrm{CO}$ is five hours, in patients breathing air, and measurements may still be of value after considerable delay.

Patients with carbon monoxide poisoning that are pregnant, that have any neurological signs or symptoms including abnormalities of balance or gait, or those with evidence of cardiovascular abnormalities including abnormal ECGs, or myocardial ischaemia/infarction, should be discussed with a hyperbaric oxygen centre. Unconscious patients should undergo early cranial imaging and may require aggressive management to control cerebral oedema.

\section{CYANIDE}

Cyanide poisoning is most frequently encountered following inhalation of smoke containing the combustion products of nitrogen containing polymers- both natural, for example, wool and silk; and synthetic, for example, polyurethane and polyacrylnitrile. Synthetic polymers are used extensively in domestic furnishings. Cyanide and carbon monoxide poisoning frequently coexist. Symington et al found that $4 \%$ of those dying in house fires had potentially lethal cyanide levels. ${ }^{9}$

Cyanide binds to ferric iron $\left(\mathrm{Fe}^{3+}\right)$ in the cytochrome a-a3 complex, inhibiting its action and blocking the final step in oxidative phosphorylation. Aerobic metabolism is halted and carbohydrate metabolism is diverted to the production of lactic acid.

Cyanide poisoning requires a high index of suspicion; signs are very hard to dissect from those of CO poisoning. Cardinal features are the presence of a profound, refractory acidosis and a massively increased anion gap. There may be very little difference between venous and arterial $\mathrm{po}_{2}$, termed arteriolisation of venous blood, because of impaired cellular respiration. Patients should be considered at high risk if they have been exposed to fumes from burning plastics, are profoundly unconscious, or have persistent myocardial ischaemia in the absence of elevated $\mathrm{COHb}$.

Rapid treatment is essential. Blood levels are useful in confirming toxicity, but therapeutic intervention is usually necessary before the level is available.

Patients should receive high concentrations of inspired oxygen. Mouth to mouth resucitation must be avoided. Specific antidotes should be administered as soon as possible (see table 1).

\section{ISOPROPANOL}

Isopropanol is an alcohol found in some nail polishes, hairsprays, antifreezes, and car screen washes. It is also found in rubbing alcohol, window cleaners, and tape and CD cleaners.

Isopropanol is absorbed rapidly from the stomach and mucus membranes. It 
causes gastric irritation, central nervous system depression, and hypotension (which is often refractory).

Isopropanol is converted to acetone by the action of alcohol dehydrogenase. Acetone is excreted through the lungs and kidneys. Ketoacidosis is uncommon in isopropanol poisoning.

Asymptomatic children should be encouraged to drink and observed for two hours. Activated charcoal does not decrease absorption. Gastric lavage and emesis are unlikely to be effective after one hour. Both are contraindicated in the presence of central nervous system depression.

Symptomatic patients require intensive support. Hypotension, secondary to peripheral vasodilatation, may require treatment with intravenous fluids and inotropes. Haemodialysis is indicated for patients not responding to supportive measures or those with a blood isopropanol concentration of more than $4 \mathrm{~g} / \mathrm{l}{ }^{10}$ Successful treatment of isopropanol poisoning has been reported using peritoneal dialysis. ${ }^{11}$ This technique does not require specialist equipment but may prove ineffective in patients with poor peritoneal perfusion caused by refractory hypotension.

\section{ETHYLENE GLYCOL}

Ethylene glycol is found in certain types of antifreeze, brake fluid, and windscreen wash. Ethylene glycol is metabolised by alcohol dehydrogenase to a variety of toxic metabolites, including glycolic and oxalic acid. Toxic effects include convulsions, coma, metabolic acidosis, hypocalcaemia, and renal failure.

Ethylene glycol is rapidly absorbed from the stomach; symptoms can occur within 30 minutes of ingestion, and attempts at gastric decontamination are likely to be unproductive.

Asymptomatic children should have blood taken for plasma ethylene glycol estimation. Blood gases, serum calcium, electrolytes, and renal function should also be measured. Symptoms of severe poisoning can occur rapidly and, even in their absence, treatment is indicated if ingestion is likely. Ethanol competes with ethylene glycol binding at the catalytic site of alcohol dehydrogenase. An oral loading dose of ethanol $(2 \mathrm{ml} / \mathrm{kg}$ of $40 \%$ ethanol over 30 minutes; most spirits match this criteria) will delay toxic effects until blood results are available. All children receiving this treatment will require admission for overnight blood sugar estimation and observation.

Symptomatic children, or those with a plasma ethylene glycol level of more than $200 \mathrm{mg} / \mathrm{l}(3.2 \mathrm{mmol} / \mathrm{l})$, should receive intravenous fomepizole ( 15 $\mathrm{mg} / \mathrm{kg}$ over 30 minutes). Fomepizole is a competitive alcohol dehydrogenase antagonist. Its use replaces intravenous ethanol therapy, the disadvantages of which include CNS depression and hypoglycaemia. ${ }^{12}$ Where fomepizole is not available, ethanol remains an alternative treatment $(7.5 \mathrm{ml} / \mathrm{kg} 10 \%$ ethanol in 5\% dextrose over 30 minutes, then $1.65 \mathrm{ml} / \mathrm{kg} / \mathrm{h}$ of $5 \%$ ethanol to keep blood level at $1-1.5 \mathrm{~g} / \mathrm{l})$. Fomepizole should be given every 12 hours $(10 \mathrm{mg} / \mathrm{kg}$ for four doses, then $15 \mathrm{mg} / \mathrm{kg}$ ) until the plasma ethylene glycol level falls below $200 \mathrm{mg} / \mathrm{l}$. Patients require regular measurements of blood gases, renal function, and plasma calcium. Patients with renal failure or resistant metabolic acidosis will require haemodialysis. ${ }^{13}$ Fomepizole is dialysed and the dosage interval should be decreased on dialysis.

\section{METHANOL}

Methanol is also found in certain antifreezes and windscreen washes. Methanol is metabolised by alcohol dehydrogenase to formaldehyde, which in turn is converted to formate. Toxic effects include severe abdominal pain, retinal toxicity, acidosis, convulsions, and coma.

Methanol poisoning can be managed in a similar manner to ethylene glycol poisoning, both ethanol and fomepizole inhibiting its metabolism. There are, however, a few important differences. Severe symptoms of methanol poisoning tend to occur after hours rather than minutes. In the absence of symptoms, hypocalcaemia, acidosis, or renal dysfunction, treatment can be withheld pending the plasma methanol and formate concentrations. Methanol has a considerably longer plasma half life than ethylene glycol (43 hours compared with 18 hours). ${ }^{12}$ For this reason elective haemodialysis is often necessary, to enhance elimination and reduce the duration of therapy.

Methylated spirit does not contain sufficient methanol to cause toxicity Adverse effects are related to its ethanol content alone.

\section{ECSTASY}

Ecstasy, 3,4-methylenedioxymethamphetamine or MDMA, is an amphetamine derivative. In common with amphetamines, it produces locomotor stimulation, euphoria, excitement, and stereotyped behaviour. In addition, Ecstasy has psychotomimetic effects, altering perception and mood. The mechanism of action of Ecstasy is uncertain but appears to relate to increased 5-hydroxytryptamine (5-HT) release accompanied by a decreased 5-HT uptake by nerve terminals. ${ }^{7}$

Ecstasy is widely available as a drug of abuse. Ecstasy is not addictive as tolerance develops rapidly to its positive effects, while negative effects are exacerbated by large doses or frequent use.
There is concern over the apparent severe idiosyncratic reactions to Ecstasy. These effects can occur following doses that have previously been well tolerated. Reactions include coma, convulsions, arrhythmias, malignant hyperthermia, rhabdomyolysis, hypertension, and multiorgan failure.

Asymptomatic children should receive activated charcoal if poisoning has occurred within one hour. Blood pressure, temperature, and ECG monitoring are advisable. Blood levels can be performed to confirm exposure. In the absence of symptoms patients can be discharged after 24 hours observation, provided that adequate parental supervision is available. Children with symptoms require a minimum of 48 hours observation. Adverse effects, related to psychomotor stimulation or hallucination, require only supportive treatment. These effects usually last for 4-6 hours, but can occur for up to 48 hours depending on the quantity of Ecstasy consumed.

Patients with signs of cardiac or central nervous system toxicity require admission to intensive care. Careful monitoring of haematological and biochemical parameters is essential. Hyperthermia may respond to simple cooling measures but, should these fail, patients should receive dantrolene $1 \mathrm{mg} / \mathrm{kg}$ intravenously over 10-15 minutes. If there is no response this dose may be repeated at 15 minute intervals to a cumulative maximum of $10 \mathrm{mg} / \mathrm{kg}$ in 24 hours. Hypertension responds to labetalol. Convulsions and agitation should be treated with benzodiazepines. Chlorpromazine and haloperidol are best avoided as they may decrease seizure threshold. Vigorous fluid replacement is often necessary to rehydrate patients, promote a diuresis to clear myoglobin, and allow for increased insensible losses. However, care is necessary as self induced water intoxication has been described in these patients. Fluid therapy should be guided by regular blood tests and invasive monitoring.

\section{LSD}

Lysergic acid diethylamide (LSD), a 5-HT agonist, is a potent hallucinogen. It is rapidly absorbed and has a short duration of action. Symptomatic children should be reassured. Gastric decontamination is not indicated. Sedation with phenothiazines should be avoided as they decrease the threshold for convulsions. In very rare instances, LSD can be associated with malignant hyperthermia.

LSD is poorly absorbed through the skin; "LSD laced" transfer tattoos are an urban myth.

\section{COCAINE}

Cocaine is a psychomotor stimulant. It acts by inhibiting the uptake of 
dopamine, noradrenaline, and 5-HT from synapses. Cocaine is supplied as a salt or, more recently, as a free base ("crack"). Crack cocaine is favoured by addicts as it can be smoked, producing an effect equivalent to intravenous injection of the salt.

In acute overdose, both forms can be equally hazardous. Effects include agitation, hallucinations, convulsions, hypertension, myocardial ischaemia, and cerebral infarction. Cardiac arrhythmias are a particular danger. ${ }^{14-16}$

Asymptomatic children should receive activated charcoal if ingestion has occurred within one hour. Blood pressure and ECG monitoring should be undertaken.

Symptomatic children require intensive monitoring. Agitation, hallucinations, and convulsions can be controlled with benzodiazepines. Acidosis exacerbates cardiac toxicity and should be corrected; resistant arrhythmias may respond to calcium antagonists. ${ }^{17}$ Hypertension and chest pain can be treated with diazepam, nitrates, and calcium antagonists. Drugs acting on noradrenergic or dopaminergic systems, for example, $\beta$ blockers or phenothiazines, should be avoided because of the risk of unanticipated interactions. Anticoagulation and thrombolysis should be considered if myocardial infarction occurs.

\section{NICOTINE}

Nicotine is a toxic alkaloid found in a range of plants, most notably tobacco. Nicotine is also used as a pesticide. Nicotine stimulates nicotinic acetylcholine receptors throughout the central and autonomic nervous systems. In severe poisoning confusion and convulsions are accompanied by arrhythmias and parasympathetic over stimulation (see organophosphate poisoning).

Children most frequently encounter nicotine in the form of cigarette or cigarette butt ingestion. Vomiting is the commonest symptom following cigarette ingestion and tends to limit toxicity. Asymptomatic children who have ingested less than one cigarette or two cigarette butts should be observed for two hours. No specific treatment is required. Children who have consumed more than this amount, should receive activated charcoal.

\section{ISONIAZID}

Isoniazid is widely used in the treatment of tuberculosis. In overdose, isoniazid reacts with pyridoxine to form a compound which is rapidly excreted in the urine. Pyridoxine is necessary for the production of the inhibitory neurotransmitter $\gamma$-aminobutyric acid (GABA). A deficiency of GABA results in intractable seizures, resistant to most conventional anticonvulsant therapies.

The effects of isoniazid toxicity appear within two hours of ingestion. Neurological symptoms predominate and seizures are likely if more than $30 \mathrm{mg} / \mathrm{kg}$ has been ingested. Lactic acidosis, hyperglycaemia, hypokalaemia, and ketonuria are common.

Asymptomatic children should receive activated charcoal. Symptomatic patients should receive intravenous pyridoxine-at a dose equivalent to the amount of isoniazid ingested in milligrams. This dose may be repeated at 10 minute intervals to control seizures. ${ }^{18}$ Aggressive supportive treatment may be required. Urinary alkalinisation ${ }^{19}$ or haemodialysis ${ }^{20}$ enhance isoniazid clearance and may be considered in patients who fail to respond.

\section{SEEKING FURTHER ADVICE}

Specific, expert advice on all aspects of poisoning is available to medical professionals in the United Kingdom via the National Poisons Information Service (NPIS). The regional centres that make up this service have recently introduced a single national enquiry number: 08706006266.

A wide range of easily accessible and highly practical advice is available through the NPIS website. ${ }^{21}$ This free service is restricted to medical professionals. On line registration is available at http://www.spib.axl.co.uk/toxbase/.

\section{Arch Dis Child 2002;87:407-410}

\section{Authors' affiliations}

M F Riordan, Department of Pediatrics, Yale University Medical School, USA

G W Rylance, Department of General

Paediatrics, Royal Victoria Infirmary, Newcastle upon Tyne, UK

K Berry, Accident and Emergency Department, Birmingham Children's Hospital, Birmingham, UK
Correspondence to: Dr K Berry, Accident and Emergency Department, Birmingham Children's Hospital, Steelhouse Lane, Birmingham B4 6NH, UK;

kathleen.berry@bhamchildrens.wmids.nhs.uk

\section{REFERENCES}

1 Bates N, Edwards N, Roper J, et al. Paediatric toxicology: handbook of poisoning in children. London: Macmillan Reference Limited, 1997.

2 Mofenson HC, Caraccio TR, Schauben J. Poisoning by antidysrhythmic drugs. Pediatr Clin North Am 1986;33:723-38.

3 Vale JA, Krenzelok EP, Barceloux GD. Position statement and practice guidelines on the use of multi-dose activated charcoal in the treatment of acute poisoning. American Academy of Clinical Toxicology; European Association of Poisons Centres and Clinical Toxicologists. J Toxicol Clin Toxicol 1999;37:731-51.

4 Commerford PJ, Lloyd EA. Arrhythmias in patients with drug toxicity, electrolyte, and endocrine disturbances. Med Clin North Am 1984;68: 1051-79.

5 Quadrani DA, Spiller HA, Widder P. Five year retrospective evaluation of sulfonylured ingestion in children. J Toxicol Clin Toxicol 1996;34:267-70.

6 McLaughlin SA, Crandall CS, McKinney PE. Octreotide: an antidote for sulfonylurea-induced hypoglycemia. Ann sulfonylurea-induced hypoglyc
Emerg Med 2000;36:133-8.

7 Rang, Dale, Ritter. Pharmacology, 4th edn. Edinburgh: Churchill Livingstone, 1999

8 Department of Trade and Industry. Home and Leisure Accident Surveillance System. 1978-1997. Personal communication, 1999.

9 Symington IS, Anderson RA, Thomson I, et al. Cyanide exposure in fires. Lancet 1978;2:91-2.

10 Lacouture PG, Wason S, Abrams A, et al. Acute isopropyl alcohol intoxication. Diagnosis and management. Am J Med 1983;75:680-6.

11 Mecikalski MB, Depner TA. Peritoneal dialysis for isopropanol poisoning. West J dialysis for isopropanol
Med 1982;137:322-4.

12 Tenenbein $M$. Recent advancements in pediatric toxicology. Pediatr Clin North Am 1999;46:1179-88, vii.

13 Watson WA. Ethylene glycol toxicity: closing in on rational, evidence-based treatment [editorial; comment]. Ann Emerg Med 2000;36:139-41.

14 Wang RY. pH-dependent cocaine-induced cardiotoxicity. Am J Emerg Med 1999;17:364-9

15 Billman GE. Cocaine: a review of its toxic actions on cardiac function. Crit Rev Toxicol 1995;25: 113-32.

16 Kloner RA, Hale S, Alker K, et al. The effects of acute and chronic cocaine use on the heart. of acute and chronic cocaine
Circulation 1992;85:407-19.

17 Nahas G, Trouve R, Demus JR, et al. A calcium-channel blocker as antidote to the cardiac effects of cocaine intoxication [letter] N Engl J Med 1985;313:519-20.

18 Romero JA, Kuczler FJ. Isoniazid overdose: recognition and management. Am Fam Physician 1998;57:749-52.

19 Yarbrough BE. Current management of the poisoned patient. South Med J 1988;81: 892-901.

20 Orlowski JP, Paganini EP, Pippenger CE. Treatment of a potentially lethal dose isoniazid ingestion. Ann Emerg Med 1988;17:73-6.

21 Good AM, Bateman DN. TOXBA'SE on the Internet. J Accid Emerg Med 1999;16:399 